\title{
The Association between Alcohol Consumption and Grip Strength in a Nationwide Survey
}

\author{
Kayoung Lee \\ Department of Family Medicine, Busan Paik Hospital, Inje University College of Medicine, Busan, Korea
}

\author{
Corresponding author \\ Kayoung Lee \\ Department of Family Medicine, Busan Paik \\ Hospital, Inje University College of Medicine, \\ 75 Bokji-ro, Busanjin-gu, Busan 47392, Korea \\ Tel: +82-51-890-6229 \\ Fax: +82-51-894-7554 \\ E-mail: kayoung.fmlky@gmail.com
}

Received: September 20, 2020

Revised: January 11, 2021

Accepted: January 12, 2021
Background: Handgrip strength (HGS) is an important predictor of long-term health and physical function. Studies have associated alcohol consumption with HGS but based on inconsistent findings. The Korea National Health and Nutrition Examination Survey (KNHANES) data were analyzed to examine the association between alcohol consumption levels and HGS based on sociodemographic and health-related factors. Methods: Using the 2014 to 2018 KNHANES data, alcohol consumption levels (abstinence, moderate, binge, and heavy consumption) and HGS levels (normal vs. weak) were determined in 8,556 men and 10,054 women (age, $49 \pm 16$ years). Logistic regression analyses were conducted after adjusting for sociodemographic and health-related factors and in subgroups of those factors. Results: Binge or heavy consumption was reported in $50.2 \%$ of men and $22.7 \%$ of women, and weak HGS was found in $4.6 \%$ of men and $9.9 \%$ of women. In the fully adjusted model, weak HGS was associated with binge consumption (odds ratio [OR], 0.51, 95\% confidence interval [CI], 0.37-0.71) and heavy consumption (OR, $0.37,95 \% \mathrm{Cl}, 0.22-0.63)$ in men, and moderate consumption (OR, 0.79, 95\% Cl, 0.67$0.93)$ and binge consumption $(\mathrm{OR}, 0.65,95 \% \mathrm{Cl}, 0.52-0.83)$ in women. An association between consumption levels and weak HGS was found in both sexes regardless of age (<65 vs. $\geq 65$ years), education/income level, exercise endurance level, presence/absence of co-morbid illness, weight, and the presence/absence of metabolic syndrome. Conclusions: Alcohol consumption may be inversely associated with weak HGS, regardless of sociodemographic and health-related factors using the 2014 to 2018 KNHANES data. Further prospective studies are necessary to examine the causality of the association.

Key Words: Alcohol drinking · Health behavior $\cdot$ Hand strength

\section{INTRODUCTION}

Weak muscle strength is associated with an increased risk of mortality, functional decline in older persons, and major osteoporotic fracture.[1-3] The association of muscle strength with mortality is independent of muscle mass.[1] Thus, the measurement of handgrip strength (HGS), which reflects muscle strength, has emerged as a clinically important screening tool for functional disability and early mortality.[4] The risk for weak HGS has been associated with older age,[5,6] lower education levels, low body mass index (BMI), comorbidity,[6] insufficient exercise, and inadequate nutritional status.[7] Excessive chronic alcohol consumption may also induce impairment of skeletal muscle protein metabolism.[8] Evidence for the potential adverse effects of alcohol consumption on muscle function has been

\section{Mineral Research}

This is an Open Access article distributed under the terms of the Creative Commons Attribution Non-Commercial License (https://creativecommons.org/licenses/by-nc/4.0/) which permits unrestricted non-commercial use, distribution, and reproduction in any medium, provided the origina work is properly cited.

\section{KSBMR}


found in previous studies: higher alcohol consumption at baseline was associated with a 2-year decline in muscle strength in middle-aged men,[9] and problematic consumption behavior was inversely associated with HGS in older men with diabetes mellitus.[10]

In contrast, the findings of meta-analysis studies suggested that alcohol consumption was associated with a lower risk for sarcopenia in older men,[11] and heavier alcohol consumption was associated with a lower incidence of frailty when compared with no alcohol consumption. [12] The findings of other studies also suggest an inverse association between alcohol consumption and weak HGS. $[6,13]$

Considering the associations between sociodemographic, health-related factors, and weak HGS, these factors may interact with alcohol consumption in terms of the association with weak HGS. Although sex- or comorbidity-specific associations between alcohol consumption and HGS have been evaluated, $[9,10]$ associations according to sociodemographic and health-related factors have been demonstrated less frequently.

Given the inconsistency in results, the limited number of relevant studies, and scarce information regarding interactions between sociodemographic and health-related factors and alcohol consumption related to weak HGS, a further study on the relationship between alcohol consumption and muscle strength (considering those factors) may extend previous findings.

The aim of this study was to explore the association between alcohol consumption levels and muscle strength (using HGS) while considering sociodemographic and healthrelated factors in population-based data of Korean adults.

\section{METHODS}

\section{Study subjects}

This study was performed using data obtained from the 2014 to 2018 Korea National Health and Nutrition Examination Survey (KNHANES).[14] KNHANES data can be requested through the following website: http://knhanes. cdc.go.kr. The KNHANES is a nationwide cross-sectional health survey conducted by the Korea Centers for Disease Control and Prevention for non-institutionalized South Koreans; it employs a rolling sampling design that involves a complex, stratified, and multistage probability cluster sur- vey.[15] Of 31,310 adults aged 19 to 80 years, individuals included in the present study were evaluated for complete data on alcohol use, HGS, demographic and health behavior-related characteristics, chronic illnesses, and anthropometric measurements. Individuals without available data for alcohol consumption $(\mathrm{N}=5,813), \mathrm{HGS}(\mathrm{N}=3,585)$, selfreported health status $(\mathrm{N}=3,442)$, components of metabolic syndrome $(\mathrm{N}=3,921)$, functional limitations $(\mathrm{N}=1,755)$, BMI $(N=1,543)$, smoking status $(N=2,400)$, physical activity including sedentary behavior and endurance and resistance exercise $(\mathrm{N}=8,607)$, dietary intake $(\mathrm{N}=3,831)$, education level $(N=3,658)$, income level $(N=152)$, and menopausal status (in women; $\mathrm{N}=822$ ) were excluded. The final dataset comprised complete data for 18,610 individuals (8,556 men, 10,054 women; $49.3 \pm 16.1$ years of age).

This work was categorized as an exemption category from the Institutional Review Board (IRB) of Busan Paik Hospital. The original KNHANES 2014 to 2017 were classified under the exemption category for ethical review in the Bioethics and Safety Act, and the KNHANES 2018 was approved by the IRB of the Korea Centers for Disease Control (IRB No. 2018-01-03-P-A). All participants provided written informed consent before enrollment to the original survey.

\section{Clinical and laboratory measurements}

Grip strength was evaluated 3 times per hand using a TKK 5401 digital grip-strength dynamometer (Takei Scientific Instruments Co. Ltd., Tokyo, Japan). All subjects stood with their hands at their sides. Then, each individual grasped the dynamometer with 1 hand, and 3 trials were conducted. The same procedure was repeated using the other hand. The subjects squeezed the dynamometer with maximum effort for approximately $3 \mathrm{sec}$; at least $30 \mathrm{sec}$ of a resting interval was allowed between each measurement. Weak HGS was defined as the maximally measured grip strength of the dominant hand,[16] $<26 \mathrm{~kg}$ for men and $<18 \mathrm{~kg}$ for women, based on low muscle strength criteria among the 3 criteria for sarcopenia, including low muscle mass and low physical performance recommended by the Asian Working Group for Sarcopenia.[5]

Alcohol consumption levels were categorized as abstinence (nondrinkers) or moderate consumption (up to 2 drinks per day for men and 1 drink per day for women), binge consumption (7 or more alcoholic drinks for men or 5 
or more drinks for women on the same occasion at least once per month but less than daily), or heavy consumption (binge consumption almost daily).[17] The assessment of consumption levels was based on 3 questions: "How many drinks (using standard-sized containers of beverages) do you have on a typical consumption day?", with 6 response options (none, 1-2 drinks, 3-4 drinks, 5-6 drinks, 7-9 drinks, and $\geq 10$ drinks); "How many days did you drink in the past year?", with 6 response options (none, $<1$ day per month, 1 day per month, 2-4 days per month, 2-3 days per week, and $\geq 4$ days per week); and "How many days did you consume $\geq 7$ drinks (in men) or $\geq 5$ drinks (in women) on the same occasion?", with 5 response options (never, $<1$ occasion per month, 1 occasion per month, 1 occasion per week, and almost every day). Considering that the amount of pure alcohol is about $10 \mathrm{~g}$, the approximate average daily alcohol intake was calculated by multiplying drinking frequency and amount of alcohol consumed per occasion and dividing by the number of months.

In the USA, a "standard" drink contains about $14 \mathrm{~g}$ of pure alcohol,[18] whereas in Korea, standard-sized containers of beverages contain 8 to $16 \mathrm{~g}$ of pure alcohol.[19] Therefore, binge consumption in the current study was defined as $\geq 7$ drinks in men or $\geq 5$ drinks in women instead of $\geq 5$ drinks in men or $\geq 4$ drinks in women, as suggested by the National Institute on Alcohol Abuse and Alcoholism.[20]

The drinking duration was calculated by subtracting the drinking start age from the current age using the question about the drinking start age. Weight and height were measured with the subjects wearing light clothing and no shoes. BMI was calculated by dividing weight $(\mathrm{kg})$ by squared height $(\mathrm{m})$. Then, the weight category was determined as nonobese (BMI $<25 \mathrm{~kg} / \mathrm{m}^{2}$ ) or obese (BMI $\geq 25 \mathrm{~kg} / \mathrm{m}^{2}$ ). Glucose levels were assessed using the hexokinase ultraviolet method. Triglyceride (TG) levels were assessed using a standard enzymatic method. High-density lipoprotein cholesterol (HDL-C) levels were assessed using a standard homogeneous enzymatic colorimetric method. All measurements were performed in a central certified laboratory using a 7600-210 automatic analyzer (Hitachi Ltd., Tokyo, Japan) after the subjects had fasted for at least $8 \mathrm{hr}$. Waist circumference was measured to the nearest $0.1 \mathrm{~cm}$ at the end of normal expiration at the midpoint between the lowest rib and iliac crest. Blood pressure (BP) was measured using a sphygmomanometer according to standard manual instruc- tions.[21] Metabolic syndrome was defined when at least 3 of the following components were satisfied: waist circumference $\geq 90 \mathrm{~cm}$ (for men) or $85 \mathrm{~cm}$ (for women) [22]; BP $\geq 130 / 85 \mathrm{mmHg}$ or a history of hypertension; fasting plasma glucose $\geq 5.6 \mathrm{mmol} / \mathrm{L}$ or a history of diabetes mellitus; TG $\geq 1.7 \mathrm{mmol} / \mathrm{L}$; and $\mathrm{HDL}-\mathrm{C}<1.03 \mathrm{mmol} / \mathrm{L}$ in men and $<1.29 \mathrm{mmol} / \mathrm{L}$ in women.[23]

Sociodemographic characteristics included age, sex, education level (did not graduate from high school vs. graduated from high school or above), and income level (low to low-middle vs. middle-high to high). Health-related factors included illnesses (diagnosis of cardiovascular disease, arthritis, or cancer), self-reported health status (excellent/ good/fair vs. poor/extremely poor), functional limitations, physical activity, current smoking status (yes vs. no), protein intake (adequate vs. inadequate), and menopausal status (of women). Physical activity was defined as endurance physical activity (yes vs. no for engaging in high-intensity activity for $>75 \mathrm{~min} /$ week, moderate-intensity activity for $>150 \mathrm{~min} /$ week, or a combination of both), strength exercises (yes vs. no for $\geq$ once/week), and sedentary activity (yes vs. no for sex-specific median time spent sitting).

Self-reported questionnaires were used to determine sociodemographic and health-related factors.[14] A 24-hr recall nutritional survey was used for dietary intake estimates of protein, and insufficient intake was determined using dietary reference intakes in the guidelines set for Koreans.[24]

\section{Statistical analysis}

A $\chi^{2}$ test for linear trend, or $t$-test was performed to compare alcohol consumption levels, sociodemographic factors, and health-related factors between individuals with weak HGS and those with normal HGS in each sex. A $\chi^{2}$ test for linear trend was applied to find a linear trend in the relationship of alcohol consumption levels with sociodemographic and health-related factors. Considering the sex differences in the distribution of alcohol consumption levels and HGS levels, a sex-specific logistic regression analysis was conducted for the associations of HGS (low vs. normal) with alcohol consumption levels, sociodemographic factors, and health-related factors. The Wald test was applied to identify the interactions of sociodemographic factors and health-related factors with alcohol consumption levels in the associations with HGS. A sex-specific logistic regres- 
sion analysis was also conducted for those associations according to the strata of sociodemographic factors and healthrelated factors. In these analyses, factors other than the stratum factor were adjusted. All analyses were performed using the IBM SPSS software version 24 (IBM Corp., Armonk, NY, USA).

\section{RESULTS}

In this sample of Korean adults, $50.2 \%$ of men and $22.7 \%$ of women were binge or heavy alcohol drinkers, and $4.6 \%$ of men and $9.9 \%$ of women had weak HGS. Table 1 presents comparison of alcohol drinking levels and sociodemographic and health-related characteristics between individuals with weak HGS and those with normal HGS in each sex. Compared to individuals with normal HGS, those with weak HGS were more likely to be non- or moderate drinker, have a long duration of drinking, be older, have lower income and educational levels, be less physically active, perceive them unhealthy, and have functional limitation and illnesses, and intake protein inadequately regardless of sex. Women with weak HGS were more likely to be obese and have metabolic syndrome compared to those with normal HGS, while men with weak HGS were less likely to be obese compared to their counterparts.

Men and women with high alcohol consumption tended to be younger $(P<0.01)$, have high educational levels $(P<0.01)$, perceive them healthy $(P<0.01)$, have no functional limitations $(P<0.01)$, no chronic diseases $(P<0.01)$, regular strength $(P<0.01)$ and aerobic exercise $(P<0.01)$, no metabolic syndrome $(P<0.01)$, or adequate protein intake $(P<0.01)$, while they tended to be smoker $(P<0.01)$ and have high BMI $(P<0.01)$ (Data are not shown).

Sex-specific associations of weak HGS with alcohol consumption levels, sociodemographic factors, and health-related factors are presented in Table 2. After adjusting for sociodemographic and health-related factors, the odds for weak HGS among men who engaged in binge or heavy consumption were $49 \%$ and $63 \%$ lower, respectively, whereas the odds for weak HGS among women who engaged in

Table 1. Comparison of alcohol consumption and sociodemographic and health-related characteristics by HGS status

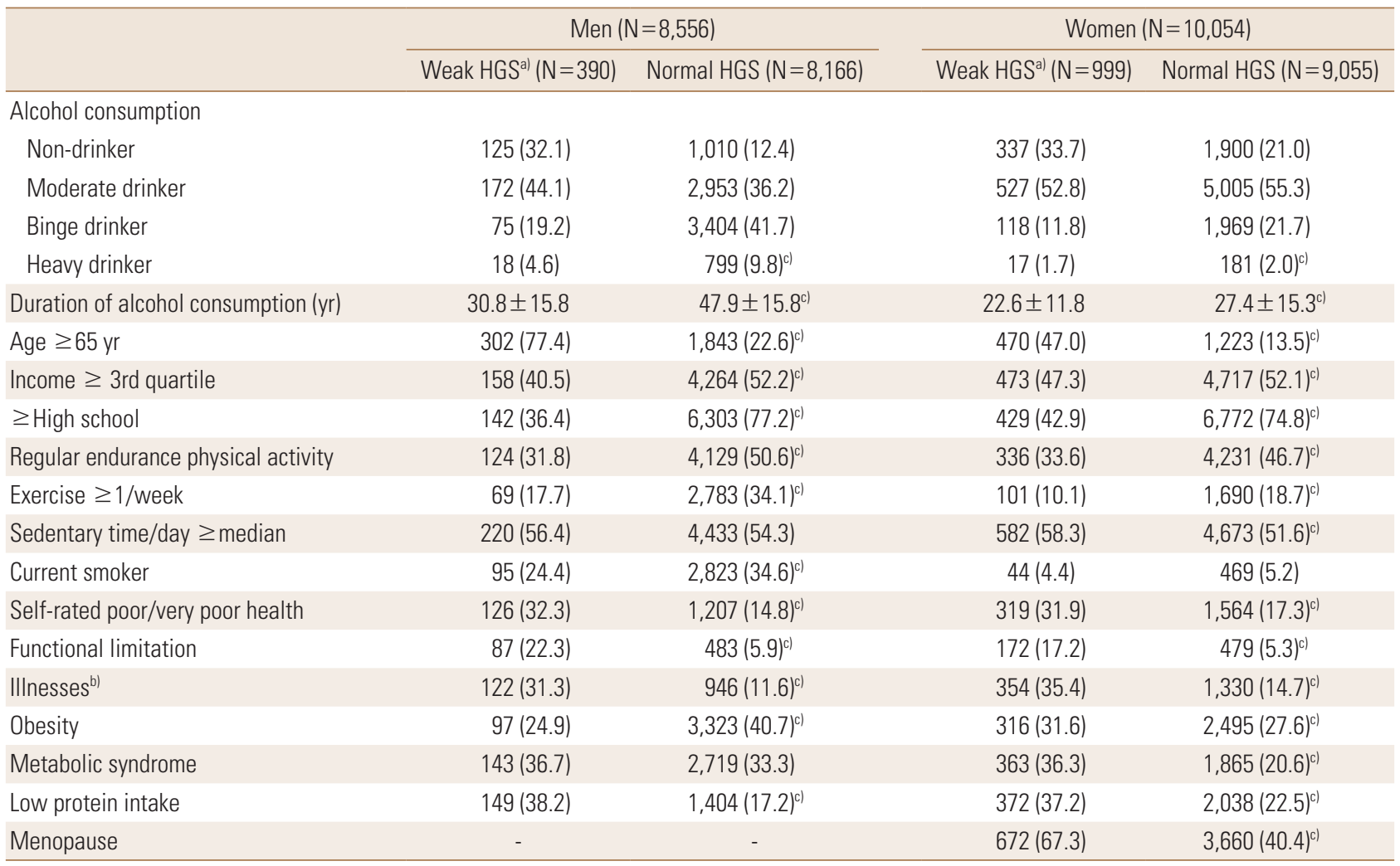

The data is presented as number (\%) or mean \pm standard deviation.

${ }^{a} \mathrm{H} H S<26 \mathrm{~kg}$ for men, $<18 \mathrm{~kg}$ for women. ${ }^{\mathrm{b}}$ Cardiovascular diseases, arthritis, and cancers. ${ }^{\mathrm{cl}} P<0.05$ using $\chi^{2}$ test for linear trend, or $t$-test. HGS, handgrip strength. 
Table 2. Sex-specific associations of weak handgrip strength ${ }^{\text {a) }}$ with alcohol consumption levels and sociodemographic and health-related factors

\begin{tabular}{|c|c|c|}
\hline & Men $(N=8,556)$ & Women $(\mathrm{N}=10,054$ \\
\hline \multicolumn{3}{|l|}{ Alcohol consumption ${ }^{\text {b) }}$} \\
\hline Non-drinker & 1.0 & 1.0 \\
\hline Moderate drinker & $0.85(0.65-1.11)$ & $0.79(0.67-0.93)$ \\
\hline Binge drinker & $0.51(0.37-0.71)$ & $0.65(0.52-0.83)$ \\
\hline Heavy drinker & $0.37(0.22-0.63)$ & $0.79(0.45-1.39)$ \\
\hline Age, $\geq 65$ yr vs. $<65$ yr & $6.15(4.61-9.21)$ & $2.84(2.35-3.44)$ \\
\hline Income, $<3$ rd quartile vs. $\geq 3$ rd quartile ${ }^{c)}$ & $1.27(1.01-1.61)$ & $1.11(0.96-1.28)$ \\
\hline Education, $<$ high school vs. $\geq$ high school ${ }^{c \mid}$ & $1.86(1.44-2.40)$ & $1.66(1.38-2.01)$ \\
\hline Endurance physical activity, irregular vs. regular ${ }^{c l}$ & $1.31(1.03-1.67)$ & $1.18(1.01-1.37)$ \\
\hline Exercise $<1 /$ week vs. $\geq 1 /$ week $^{c \mid}$ & $1.84(1.38-2.45)$ & $1.51(1.21-1.89)$ \\
\hline Sedentary time, $\geq$ median vs. $<$ median $^{c)}$ & $1.20(0.96-1.51)$ & $1.27(1.10-1.47)$ \\
\hline Current smoker, yes vs. no ${ }^{c l}$ & $0.97(0.74-1.27)$ & $0.95(0.67-1.35)$ \\
\hline Self-rated health, poor/very poor vs. fair to excellent ${ }^{\mathrm{c}}$ & $1.28(0.98-1.68)$ & $1.26(1.07-1.50)$ \\
\hline Functional limitation, presence vs. absence ${ }^{c \mid}$ & $1.92(1.41-2.62)$ & $1.67(1.34-2.09)$ \\
\hline IIInesses, presence vs. absence ${ }^{c \mid}$ & $1.05(0.81-1.36)$ & $1.20(1.01-1.42)$ \\
\hline Obesity vs. non-obesityc) & $0.55(0.42-0.73)$ & $0.74(0.63-0.88)$ \\
\hline Metabolic syndrome, presence vs. absence ${ }^{c \mid}$ & $1.07(0.83-1.38)$ & $1.16(0.97-1.39)$ \\
\hline Protein intake, inadequate vs. adequate ${ }^{c)}$ & $1.59(1.25-2.02)$ & $1.39(1.19-1.61)$ \\
\hline Menopause vs. premenopause ${ }^{c)}$ & & $0.99(0.80-1.23)$ \\
\hline
\end{tabular}

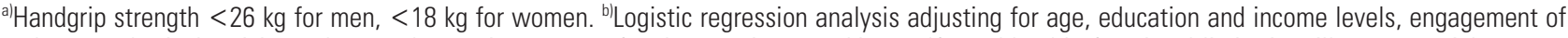
endurance physical activity and strength exercise, status of sedentary time, smoking, self-rated health, functional limitation, illnesses, weight, metabolic syndrome, adequacy of protein intake, and menopause (in women). 'Logistic regression analysis adjusting for the same confounding factors (except for that variable) and alcohol drinking levels.

moderate or binge consumption were $21 \%$ and $35 \%$ lower, respectively, compared to male or female nondrinkers. In both men and women, the odds for weak HGS increased for those who were 65 years of age or older, had low education levels, engaged in irregular endurance or resistance exercises, had functional limitations, were not obese, and had inadequate protein intake levels compared to their counterparts. Additionally, women who spent more time in a sedentary position and had poor self-reported health and illnesses had higher odds for weak HGS compared to their counterparts (Table 2).

The sex-specific associations between alcohol consumption levels and HGS in the strata of sociodemographic and health-related factors are presented in Table 3 for men and Table 4 for women. Interaction with alcohol consumption levels was significant only for strength exercise in men, while there were no significant interactions between other factors and alcohol consumption levels in either men or women. Compared to male nondrinkers, male binge-to-heavy drinkers were less likely to have weak HGS, regardless of age, income and education levels, endurance exercise lev- el, sedentary time level, smoking status, self-rated health status, illness category, weight status, and metabolic syndrome category. Compared to female nondrinkers, female moderate-to-binge drinkers were less likely to have weak HGS, regardless of age, income and education levels, regularity of endurance exercise, illness category, weight status, metabolic syndrome category, and menopausal status. However, alcohol consumption levels and weak HGS were not associated in men who performed regular strength exercises or had functional limitations or inadequate protein intake; they were also not associated in women who performed regular strength exercises, exhibited current smoking behavior, and had short sedentary times, poor self-rated health, functional limitations, or inadequate protein intake.

\section{DISCUSSION}

Based on the 2014 to 2018 KNHANES data, findings of this cross-sectional study suggest that binge-to-heavy consumption levels in men and moderate-to-binge consump- 
Table 3. The associations of weak handgrip strengthal with alcohol consumption levels according to sociodemographic and health-related factors in $\operatorname{men}(\mathrm{N}=8,556)$

\begin{tabular}{|c|c|c|c|c|}
\hline & Non-drinker & Moderate drinker & Binge drinker & Heavy drinker \\
\hline \multicolumn{5}{|l|}{$\mathrm{Age}^{\mathrm{bl}}$} \\
\hline$\geq 65 \mathrm{yr}$ & 1.0 & 0.87 (0.65-1.18) & $0.53(0.35-0.79)$ & $0.45(0.24-0.83)$ \\
\hline$<65 y r$ & 1.0 & $0.70(0.30-1.26)$ & $0.43(0.23-0.80)$ & $0.22(0.07-0.65)$ \\
\hline \multicolumn{5}{|l|}{ Income $e^{\text {b) }}$} \\
\hline$<3 r d$ quartile & 1.0 & 0.97 (0.68-1.39) & $0.61(0.39-0.93)$ & $0.28(0.14-0.59)$ \\
\hline$\geq 3$ rd quartile & 1.0 & $0.71(0.46-1.08)$ & $0.40(0.23-0.68)$ & $0.56(0.26-1.21)$ \\
\hline \multicolumn{5}{|l|}{ Education ${ }^{\text {b) }}$} \\
\hline$<$ High school & 1.0 & $0.82(0.58-1.16)$ & $0.50(0.32-0.77)$ & $0.42(0.23-0.77)$ \\
\hline$\geq$ High school & 1.0 & $0.87(0.55-1.37)$ & $0.51(0.30-0.88)$ & $0.22(0.07-0.74)$ \\
\hline \multicolumn{5}{|l|}{ Physical activity ${ }^{b)}$} \\
\hline Irregular & 1.0 & $0.89(0.64-1.23)$ & $0.52(0.34-0.79)$ & $0.46(0.25-0.86)$ \\
\hline Regular & 1.0 & $0.69(0.43-1.12)$ & $0.46(0.27-0.81)$ & $0.20(0.07-0.59)$ \\
\hline \multicolumn{5}{|l|}{ Strength exercise $e^{b, c)}$} \\
\hline$<1 /$ week & 1.0 & $0.81(0.61-1.10)$ & $0.44(0.30-0.64)$ & $0.31(0.17-0.57)$ \\
\hline$\geq 1 /$ week & 1.0 & $1.01(0.50-2.01)$ & $0.88(0.41-1.92)$ & $0.79(0.24-1.56)$ \\
\hline \multicolumn{5}{|l|}{ Sedentary time ${ }^{\mathrm{b})}$} \\
\hline$\geq$ Median & 1.0 & $0.78(0.54-1.13)$ & $0.44(0.28-0.69)$ & $0.34(0.16-0.70)$ \\
\hline$<$ Median & 1.0 & $1.00(0.66-1.51)$ & $0.63(0.39-1.04)$ & $0.44(0.20-0.99)$ \\
\hline \multicolumn{5}{|l|}{ Current smoker ${ }^{\mathrm{b})}$} \\
\hline Yes & 1.0 & $0.67(0.37-1.21)$ & $0.44(0.23-0.87)$ & $0.41(0.18-0.98)$ \\
\hline No & 1.0 & $0.89(0.66-1.21)$ & $0.52(0.36-0.77)$ & $0.31(0.15-0.63)$ \\
\hline \multicolumn{5}{|l|}{ Self-rated health ${ }^{\text {b) }}$} \\
\hline Poor/Very poor & 1.0 & $0.87(0.54-1.38)$ & $0.46(0.24-0.86)$ & $0.73(0.32-1.64)$ \\
\hline Fair/Good/Excellent & 1.0 & $0.83(0.60-1.16)$ & $0.52(0.35-0.78)$ & $0.25(0.12-0.51)$ \\
\hline \multicolumn{5}{|l|}{ Functional limitation ${ }^{\text {b) }}$} \\
\hline Presence & 1.0 & $1.36(0.75-1.48)$ & $0.77(0.35-1.70)$ & $0.52(0.17-1.60)$ \\
\hline Absence & 1.0 & $0.75(0.55-1.02)$ & $0.46(0.32-0.66)$ & $0.32(0.17-0.59)$ \\
\hline \multicolumn{5}{|l|}{ IInesses ${ }^{b)}$} \\
\hline Presence & 1.0 & $1.10(0.70-1.73)$ & $0.50(0.26-0.96)$ & $0.09(0.01-0.65)$ \\
\hline Absence & 1.0 & $0.75(0.53-1.05)$ & $0.51(0.35-0.76)$ & $0.45(0.25-0.80)$ \\
\hline \multicolumn{5}{|l|}{ Obesity ${ }^{b /}$} \\
\hline Presence & 1.0 & $0.85(0.49-1.45)$ & $0.46(0.24-0.87)$ & $0.38(0.14-1.03)$ \\
\hline Absence & 1.0 & $0.83(0.61-1.14)$ & $0.53(0.36-0.79)$ & $0.36(0.19-0.68)$ \\
\hline \multicolumn{5}{|l|}{ Metabolic syndrome ${ }^{\text {b) }}$} \\
\hline Presence & 1.0 & $0.75(0.47-1.18)$ & $0.50(0.30-0.84)$ & $0.47(0.20-1.00)$ \\
\hline Absence & 1.0 & $0.90(0.64-1.27)$ & $0.52(0.34-0.80)$ & $0.29(0.13-0.62)$ \\
\hline \multicolumn{5}{|l|}{ Protein intake $e^{\text {b) }}$} \\
\hline Inadequate & 1.0 & $1.09(0.69-1.72)$ & $0.74(0.42-1.31)$ & $0.58(0.26-1.30)$ \\
\hline Adequate & 1.0 & $0.73(0.52-1.02)$ & $0.42(0.28-0.64)$ & $0.27(0.13-0.56)$ \\
\hline
\end{tabular}

The data is presented as odds ratio ( $95 \%$ confidence interval).

a) Handgrip strength $<26 \mathrm{~kg}$. b) Logistic regression analysis after adjusting for age, education, income, smoking status, endurance exercise, resistance exercise, sedentary time, self-rated health, functional limitation, chronic illness, obesity, metabolic syndrome, and protein intake (except for the stratum variable). ${ }^{\mathrm{cl}} P<0.05$ using the Wald test.

tion levels in women had inverse associations with weak HGS after adjusting for sociodemographic and health-re- lated factors. Male binge-to-heavy drinkers and female moderate-to-binge drinkers were $49 \%$ to $63 \%$ and $21 \%$ to 
Table 4. The associations of low handgrip strength ${ }^{\text {a) }}$ with alcohol consumption levels according to sociodemographic and health-related factors in women $(\mathrm{N}=10,054)$

\begin{tabular}{|c|c|c|c|c|}
\hline & Non-drinker & Moderate drinker & Binge drinker & Heavy drinker \\
\hline \multicolumn{5}{|l|}{$\mathrm{Age}^{\mathrm{b})}$} \\
\hline$\geq 65 \mathrm{yr}$ & 1.0 & $0.81(0.64-1.03)$ & $0.43(0.23-0.81)$ & $1.40(0.45-1.41)$ \\
\hline$<65 \mathrm{yr}$ & 1.0 & 0.79 (0.63-0.99) & $0.71(0.54-0.93)$ & $0.71(0.36-1.40)$ \\
\hline \multicolumn{5}{|l|}{ Income $e^{\text {bl }}$} \\
\hline$<3$ rd quartile & 1.0 & 0.80 (0.64-0.99) & $0.60(0.43-0.83)$ & $0.84(0.43-1.66)$ \\
\hline$\geq 3$ rd quartile & 1.0 & $0.78(0.62-0.98)$ & $0.72(0.51-1.01)$ & $0.65(0.22-1.87)$ \\
\hline \multicolumn{5}{|l|}{ Education ${ }^{\text {b) }}$} \\
\hline$<$ High school & 1.0 & $0.85(0.69-1.05)$ & $0.63(0.42-0.94)$ & $0.87(0.39-1.96)$ \\
\hline$\geq$ High school & 1.0 & $0.74(0.58-0.95)$ & $0.65(0.48-0.89)$ & $0.74(0.33-1.65)$ \\
\hline \multicolumn{5}{|l|}{ Physical activity ${ }^{b}$} \\
\hline Irregular & 1.0 & $0.98(0.80-1.19)$ & $0.69(0.50-0.95)$ & $0.87(0.42-1.80)$ \\
\hline Regular & 1.0 & $0.54(0.42-0.71)$ & $0.56(0.39-0.80)$ & $0.80(0.33-1.95)$ \\
\hline \multicolumn{5}{|l|}{ Strength exercise ${ }^{b)}$} \\
\hline$<1 /$ week & 1.0 & $0.80(0.68-0.95)$ & $0.65(0.50-0.85)$ & $0.90(0.50-1.64)$ \\
\hline$\geq 1 /$ week & 1.0 & $0.72(0.44-1.18)$ & $0.65(0.34-1.24)$ & $0.31(0.04-2.44)$ \\
\hline \multicolumn{5}{|l|}{ Sedentary time ${ }^{\text {b) }}$} \\
\hline$\geq$ Median & 1.0 & $0.74(0.60-0.91)$ & $0.61(0.44-0.85)$ & $0.70(0.32-1.51)$ \\
\hline$<$ Median & 1.0 & 0.89 (0.69-1.13) & $0.73(0.51-1.05)$ & $0.93(0.40-2.14)$ \\
\hline \multicolumn{5}{|l|}{ Current smokerb) } \\
\hline Yes & 1.0 & $2.26(0.69-7.34)$ & $2.45(0.68-8.87)$ & $0.87(0.14-5.49)$ \\
\hline No & 1.0 & $0.78(0.66-0.91)$ & $0.61(0.48-0.79)$ & $1.02(0.56-1.87)$ \\
\hline \multicolumn{5}{|l|}{ Self-rated health ${ }^{\text {b) }}$} \\
\hline Poor/Very poor & 1.0 & $0.85(0.64-1.13)$ & $0.64(0.38-1.09)$ & $0.95(0.27-3.39)$ \\
\hline Fair/Good/Excellent & 1.0 & $0.77(0.63-0.93)$ & $0.64(0.43-0.85)$ & $0.74(0.39-1.40)$ \\
\hline \multicolumn{5}{|l|}{ Functional limitation ${ }^{\text {b) }}$} \\
\hline Presence & 1.0 & $0.96(0.64-1.44)$ & $0.97(0.45-2.07)$ & - \\
\hline Absence & 1.0 & $0.76(0.64-0.91)$ & $0.63(0.49-0.81)$ & $0.86(0.49-1.52)$ \\
\hline \multicolumn{5}{|l|}{ Illnesses ${ }^{b)}$} \\
\hline Presence & 1.0 & $0.86(0.67-1.12)$ & $0.39(0.21-0.71)$ & $0.53(0.11-2.43)$ \\
\hline Absence & 1.0 & $0.76(0.62-0.93)$ & $0.71(0.54-0.93)$ & $0.83(0.45-1.53)$ \\
\hline \multicolumn{5}{|l|}{ Obesity ${ }^{b)}$} \\
\hline Presence & 1.0 & $0.77(0.58-1.02)$ & $0.44(0.27-0.72)$ & $1.02(0.43-2.43)$ \\
\hline Absence & 1.0 & $0.80(0.66-0.98)$ & $0.74(0.56-0.98)$ & $0.65(0.31-1.39)$ \\
\hline \multicolumn{5}{|l|}{ Metabolic syndrome ${ }^{\text {b) }}$} \\
\hline Presence & 1.0 & $0.78(0.60-1.01)$ & $0.52(0.31-0.86)$ & $0.99(0.41-2.39)$ \\
\hline Absence & 1.0 & 0.81 (0.66-0.99) & $0.70(0.53-0.93)$ & $0.69(0.32-1.46)$ \\
\hline \multicolumn{5}{|l|}{ Protein intake $e^{\text {b) }}$} \\
\hline Inadequate & 1.0 & $0.95(0.72-1.25)$ & $0.56(0.35-0.89)$ & $0.42(0.12-1.44)$ \\
\hline Adequate & 1.0 & $0.72(0.59-0.87)$ & $0.68(0.51-0.90)$ & $0.97(0.51-1.82)$ \\
\hline \multicolumn{5}{|l|}{ Menopausal status ${ }^{\text {b) }}$} \\
\hline Premenopause & 1.0 & $0.67(0.50-0.90)$ & $0.71(0.51-0.99)$ & $0.56(0.23-1.35)$ \\
\hline Postmenopause & 1.0 & $0.86(0.71-1.04)$ & $0.48(0.32-0.71)$ & $1.19(0.55-2.58)$ \\
\hline
\end{tabular}

The data is presented as odds ratio ( $95 \%$ confidence interval).

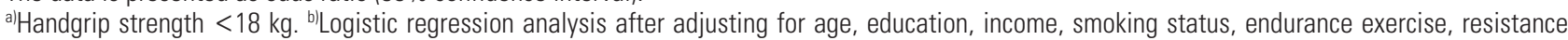
exercise, sedentary time, self-rated health, functional limitation, chronic illness, obesity, metabolic syndrome, protein intake, and menopausal status in each stratum (except for the stratum variable itself). 
$35 \%$ less likely to have weak HGS compared to male or female nondrinkers, respectively. In both sexes, these associations were consistent in the strata of age, education and income levels, endurance exercise level, comorbid illness, weight status, and comorbid metabolic syndrome. In men, an inverse association between consumption levels and weak HGS was also found in the strata of sedentary time levels, smoking status, and self-rated health-and in women, in the stratum of menopausal status. However, alcohol consumption had a significant interaction with strength exercise in men. In other words, in men who performed strength exercise less than once a week, the odds ratio of weak grip strength decreased as the amount of alcohol increased, whereas in those who performed strength exercise more than once a week, there was no relationship between the amount of alcohol and grip strength.

Stating whether alcohol consumption is a risk factor or a protective factor for sarcopenia, frailty, or weak HGS is ambiguous. In a meta-analysis using 13 cross-sectional studies in which alcohol drinking group was defined as those who consumed alcohol regardless of the period or intensity, the risk for sarcopenia based on the measurement of muscle mass was lower in men and women who consumed alcohol compared with nondrinkers.[11] In another metaanalysis using 4 prospective studies in which alcohol consumption was evaluated using quantity or frequency, heavier alcohol consumption was associated with $39 \%$ to $56 \%$ lower incidence of frailty compared with the frailty status of nondrinkers.[12] In a previous study among white older women, nondrinkers had poorer performance measures that included muscle strength using grip strength, agility and coordination, gait and balance, and self-reported functional status, compared with current moderate drinkers. [13] A cross-sectional study involving a Chinese population indicated that current drinkers were associated with better performance of HGS and usual gait speed compared to nondrinkers.[6]

In contrast, a recent longitudinal study in which drinking levels were categorized into sex-specific quartiles based on calculation of drinking frequency and the type of alcoholic beverages suggested that high alcohol consumption (when compared with low and medium alcohol consumption) was more likely to be associated with a decline in HGS after 2 years in Japanese men, but not in women.[9] A crosssectional study also demonstrated an association between problem drinking assessed using the Alcohol Use Disorders Identification Test (AUDIT) questionnaire and a decline in HGS among older men with diabetes mellitus, but not in women with type 2 diabetes mellitus and older individuals without diabetes mellitus.[10] The discrepancy between study findings may probably be explained by differences in the sociodemographic and health-related characteristics of the subjects, the measurement of alcohol consumption, and the adjustment for confounding factors. However, findings of the current study indicated that the inverse association between alcohol consumption and weak HGS was consistent in subgroups of sociodemographic and health-related factors, regardless of adjustments for those factors. Therefore, the current findings can be differentiated from previous studies in that it tried to exclude the residual influence of the sociodemographic characteristics and healthrelated characteristics on the relationship between alcohol consumption and muscle strength.

The underlying mechanisms for a lower risk of weak HGS among drinkers compared with nondrinkers are not clear. Excessive chronic alcohol consumption has adverse effects on multiple organ systems,[25] and alcoholic myopathy has been reported among patients who misuse alcohol.[8] Chronic alcohol consumption may induce dysregulation of skeletal muscle protein metabolism by inhibiting global protein synthesis under basal conditions and response to several anabolic stimuli (e.g., growth factors, nutrients, and muscle contraction). Potential inhibiting mechanisms include reduction in the mammalian/mechanistic target of rapamycin (mTOR) kinase activity and an exacerbation of the decrement in mTOR and/or muscle protein synthesis present in other catabolic states.[8] Considering the mechanisms of alcohol consumption related to muscle atrophy and the present cross-sectional study, the current findings may be explained by reverse causation or uncaptured residual confounding factors. Thus, those with weak HGS might be more likely to be nondrinkers because of poor health (sick quitters).[12] In the current study, individuals with high alcohol consumption tended to have positive sociodemographic characteristics, better health, functional, and lifestyle factors for HGS. Those positive factors for HGS may have residual effects despite adjustment of those factors and explain the inverse association between alcohol consumption levels and HGS. In addition, unmeasured confounding factors (i.e., jobs, work, eating patterns, and 
social networks) might affect both alcohol consumption and HGS.

Findings of the current study also demonstrate well-established sociodemographic and health-related factors related to weak HGS. The risk factors such as older age, lower education level, low BMI, and comorbidity for weak HGS found in the current study are in agreement with a previous study in Chinese.[6] In addition, the current findings highlight the positive effects of exercise and/or nutritional intervention for improving sarcopenia in older people.[7]

In terms of clinical implications, the current findings may not support the reduction of alcohol consumption as an approach to reducing cases of weak HGS. Considering the fact that this study is a cross-sectional study before inferring causality by observing alcohol intake and grip strength prospectively, caution may be necessary to make recommendations that alcohol intake has a positive effect on grip strength.

Other limitations, except for reverse causation and residual confounding factors, should be noted. The estimated alcohol consumption levels based on self-reporting might have had a recall bias and induced misclassifications of alcohol drinking levels. The current results may not be extrapolated to other populations. Besides, the relationship between the precise cumulative alcohol consumption and HGS is not possible to examine because the KNHANES data did not provide average drinking units over time. Although the current study shows the relationship between alcohol consumption and muscle strength, the current findings may not be extended to the relationship with low muscle mass, another definition of sarcopenia.

In conclusion, findings of this population-based study indicated negative associations of weak HGS with bingeto-heavy drinking levels in men and moderate-to-binge drinking levels in women, and the findings were consistent in most sociodemographic and health-related factor-specific analyses. Considering the possibilities of reverse causation and bias, further well-designed prospective studies would clarify causality between alcohol consumption and muscle strength.

\section{DECLARATIONS}

\section{Funding}

This work was supported by the Ministry of Education of the Republic of Korea and the National Research Foundation of Korea (NRF-2020S1A5C2A02092454).

\section{Ethics approval and consent to participate}

The original KNHANES 2014-2017 were classified under the exemption category for ethical review in the Bioethics and Safety Act, and the KNHANES 2018 was approved by the IRB of the Korea Centers for Disease Control (IRB no. 2018-01-03-P-A). All patients provided written informed consent prior to enrollment.

\section{Conflict of interest}

No potential conflict of interest relevant to this article was reported.

\section{ORCID}

Kayoung Lee https://orcid.org/0000-0002-2816-554X

\section{REFERENCES}

1. Li R, Xia J, Zhang XI, et al. Associations of muscle mass and strength with all-cause mortality among US older adults. Med Sci Sports Exerc 2018;50:458-67. http://dx.doi.org/ 10.1249/mss.0000000000001448.

2. Schaap LA, Koster A, Visser M. Adiposity, muscle mass, and muscle strength in relation to functional decline in older persons. Epidemiol Rev 2013;35:51-65. http://dx.doi.org/ 10.1093/epirev/mxs006.

3. Lee SH, Gong HS. Measurement and interpretation of handgrip strength for research on sarcopenia and osteoporosis. J Bone Metab 2020;27:85-96. http://dx.doi.org/10.11005/ jbm.2020.27.2.85.

4. Shaughnessy KA, Hackney KJ, Clark BC, et al. A narrative review of handgrip strength and cognitive functioning: Bringing a new characteristic to muscle memory. J Alzheimers Dis 2020;73:1265-78. http://dx.doi.org/10.3233/ jad-190856.

5. Chen LK, Woo J, Assantachai P, et al. Asian Working Group for sarcopenia: 2019 consensus update on sarcopenia diagnosis and treatment. J Am Med Dir Assoc 2020;21:3007.e2. http://dx.doi.org/10.1016/j.jamda.2019.12.012.

6. Xu HQ, Shi JP, Shen C, et al. Sarcopenia-related features and factors associated with low muscle mass, weak muscle strength, and reduced function in Chinese rural residents: a cross-sectional study. Arch Osteoporos 2018;14:2. 
http://dx.doi.org/10.1007/s11657-018-0545-2.

7. Yoshimura $Y$, Wakabayashi $H$, Yamada $M$, et al. Interventions for treating sarcopenia: A systematic review and meta-analysis of randomized controlled studies. J Am Med Dir Assoc 2017;18:553.e1-.e16. http://dx.doi.org/10.1016/ j.jamda.2017.03.019.

8. Steiner JL, Lang CH. Dysregulation of skeletal muscle protein metabolism by alcohol. Am J Physiol Endocrinol Metab 2015;308:E699-712. http://dx.doi.org/10.1152/ajpendo. 00006.2015

9. Cui Y, Huang C, Momma $\mathrm{H}$, et al. The longitudinal association between alcohol consumption and muscle strength: A population-based prospective study. J Musculoskelet Neuronal Interact 2019;19:294-9.

10. Buchmann N, Spira D, König M, et al. Problematic drinking in the old and its association with muscle mass and muscle function in type II diabetes. Sci Rep 2019;9:12005. http: //dx.doi.org/10.1038/s41598-019-47787-0.

11. Steffl M, Bohannon RW, Petr M, et al. Alcohol consumption as a risk factor for sarcopenia - a meta-analysis. BMC Geriatr 2016;16:99. http://dx.doi.org/10.1186/s12877-0160270-x.

12. Kojima G, Liljas A, lliffe $S$, et al. A systematic review and meta-analysis of prospective associations between alcohol consumption and incident frailty. Age Ageing 2018;47: 26-34. http://dx.doi.org/10.1093/ageing/afx086.

13. Nelson HD, Nevitt MC, Scott JC, et al. Smoking, alcohol, and neuromuscular and physical function of older women. Study of Osteoporotic Fractures Research Group. Jama 1994;272:1825-31. http://dx.doi.org/10.1001/jama.1994. 03520230035035.

14. Ministry of Health \& Welfare, Korea Centers for Disease Control \& Prevention. The seventh Korea national health and nutrition examination survey (KNHANES VII-3). Osong: Korea Center for Disease Control \& Prevention; 2018.

15. Kweon S, Kim Y, Jang MJ, et al. Data resource profile: the Korea National Health and Nutrition Examination Survey (KNHANES). Int J Epidemiol 2014;43:69-77. http://dx.doi. org/10.1093/ije/dyt228.

16. Roberts HC, Denison HJ, Martin HJ, et al. A review of the measurement of grip strength in clinical and epidemiological studies: towards a standardised approach. Age Ageing 2011;40:423-9. http://dx.doi.org/10.1093/ageing/ afr051.

17. U.S. Department of Health and Human Services, U.S. De- partment of Agriculture. 2015-2020 Dietary guidelines for Americans. 8th ed. Washington, DC: U.S. Department of Health and Human Services, U.S. Department of Agriculture; 2015.

18. National Institutes of Health. What's a "standard" drink? [cited by 2020 May 22]. Available from: https://www.rethinkingdrinking.niaaa.nih.gov/How-much-is-too-much/ What-counts-as-a-drink/Whats-A-Standard-Drink.aspx

19. Korea Centers for Disease Control and Prevention, SNU R\&D Foundation. National epidemiological survey on alcohol use and problems. 2016 [cited by 2020 May 22]. Available from: http://health.cdc.go.kr/health/mobileweb/ content/group_view.jsp?CID=K1C21DS1R0

20. National Institute on Alcohol Abuse and Alcoholism. Drinking levels defined. 2020 [cited by 2020 May 22]. Available from: https://www.niaaa.nih.gov/alcohol-health/overviewalcohol-consumption/moderate-binge-drinking

21. Ministry of Health \& Welfare, Korea Centers for Disease Control \& Prevention. Procedural manual. The sixth Korea national health and nutrition examination survey $(\mathrm{KNH}-$ ANES VI-1). Osong: Korean Centers for Disease Control and Prevention; 2013.

22. Lee SY, Park HS, Kim DJ, et al. Appropriate waist circumference cutoff points for central obesity in Korean adults. Diabetes Res Clin Pract 2007;75:72-80. http://dx.doi.org/ 10.1016/j.diabres.2006.04.013.

23. Alberti KG, Eckel RH, Grundy SM, et al. Harmonizing the metabolic syndrome: a joint interim statement of the International Diabetes Federation Task Force on Epidemiology and Prevention; National Heart, Lung, and Blood Institute; American Heart Association; World Heart Federation; International Atherosclerosis Society; and International Association for the Study of Obesity. Circulation 2009; 120:1640-5. http://dx.doi.org/10.1161/circulationaha.109. 192644.

24. Ministry of Health and Welfare, The Korean Nutrition Society. Dietary reference intakes for Koreans 2015. 2015 [cited by 2019 Jul 2]. Available from: http://www.nibiohn. go.jp/eiken/kenkounippon21/download_files/foreign/ foreign_kijun_korea.pdf

25. Jayasekara H, English DR, Room R, et al. Alcohol consumption over time and risk of death: a systematic review and meta-analysis. Am J Epidemiol 2014;179:1049-59. http:// dx.doi.org/10.1093/aje/kwu028. 